\section{Response of central serous chorioretinopathy evaluated by multimodal retinal imaging}

R Sacconi ${ }^{1,2}$, G Baldin 1 , A Carnevali 1,3 , L Querques, ${ }^{1,4}$, A Rabiolo ${ }^{1}$, G Marchini², F Bandello ${ }^{1}$ and $G$ Querques ${ }^{1}$
'Department of Ophthalmology, University Vita-Salute, IRCCS Ospedale San Raffaele, Milan, Italy

${ }^{2}$ Department of Ophthalmology, University of Verona, University Hospital of Verona, Verona, Italy

${ }^{3}$ Department of Ophthalmology, University of 'Magna Graecia', Catanzaro, Italy

${ }^{4}$ G.B. Bietti FoundationIRCCS, Rome, Italy

Correspondence:

G Querques, Department of Ophthalmology, University Vita-Salute, IRCCS Ospedale San Raffaele, Via Olgettina 60, Milan 20132, Italy

Tel: +39 226434 004; Fax: +39226433643. E-mail: giuseppe.querques@ hotmail.it

Received: 9 August 2017 Accepted in revised form: 15 September 2017 Published online: 5 January 2018

\begin{abstract}
Purpose To identify predictive biomarkers of treatment outcomes by multimodal retinal imaging in patients affected by central serous chorioretinopathy (CSC).

Patients and methods In this interventional non-randomized clinical study, 27 treatmentnaive CSC patients were prospectively enrolled and treated with oral eplerenone for 513 weeks. Primary outcomes included presence of pathological findings on indocyaine green angiography (ICGA), structural optical coherence tomography (OCT) and OCTangiography (OCT-A) at baseline associated with different response to the treatment. Results A total of 29 eyes of 27 patients (2 females, 25 males) met the inclusion criteria and were included in the study (mean age was $45 \pm 7$ years). Mean CSC duration at baseline was $13.5 \pm 4.4$ weeks. After a mean of $\mathbf{1 0 . 5}$ weeks of treatment, mean central macular thickness significantly reduced $(P<0.001)$, and mean best-corrected visual acuity improved $(P<0.001)$. Seventeen eyes $(61 \%)$ demonstrated total reabsorption of subretinal fluid on structural OCT, five eyes $(18 \%)$ presented a partial response to eplerenone therapy and six eyes $(21 \%)$ showed no response. The complete response to the treatment was associated with absence of CNV at OCT-A and the presence of hotspot at ICGA $(P<0.001$ and $P=0.002$, respectively). None of eight eyes with $\mathrm{CNV}$ in OCT-A imaging had a complete response to eplerenone and none of three eyes without hotspot at ICGA showed a complete response to the treatment.
\end{abstract}

Conclusions Multimodal retinal imaging allowed us to propose predictive biomarkers (ie, absence of CNV on OCT-A and presence of hotspot on ICGA) for treatment outcomes.
Eye (2018) 32, 734-742; doi:10.1038/eye.2017.295; published online 5 January 2018

\section{Introduction}

Central serous chorioretinopathy (CSC) is one of the most common causes of central vision loss and typically affects young adult men, with an incidence of $\sim 1 / 10000 .{ }^{1}$ Acute episodes are characterized by macular serous retinal detachment from the retinal pigment epithelium (RPE) and generally resolve spontaneously in the first months. Nevertheless, in $5-10 \%$ of cases the disorder may progress to chronic CSC, which is associated with persistent serous detachment, widespread RPE alterations and cystoid retinal degeneration. Some patients can also develop choroidal neovascularization $(\mathrm{CNV})$, which leads to severe loss in visual acuity. ${ }^{3}$

Although the pathogenesis of CSC is not fully understood, many authors ascribed it to increased choroidal vascular permeability with a subsequent raise of hydrostatic pressure and alteration of the overlying RPE. ${ }^{4-6}$ Indocyanine green angiography (ICGA) demonstrated the choroidal vascular hyperpermeability in affected and contralateral eyes, and, more recently, the use of enhanced depth imaging optical coherence tomography (OCT) revealed an increased choroidal thickness in eyes affected by CSC. ${ }^{7-10}$

Several studies well documented the role of corticosteroids in CSC, referring to both glucocorticoid (cortisol) and mineralocorticoid (aldosterone) hormones. ${ }^{11-15}$ Zhao and associates ${ }^{16}$ reported that intravitreous injection of aldosterone, a specific mineralocorticoid receptor (MR) activator, induced choroidal vessel dilation and leakage in rodents' eyes. As 
MR antagonists reversed this effect, two specific MR antagonists (spironolactone and eplerenone) were used in the treatment of CSC with promising results in bestcorrected visual acuity (BCVA) and central macular thickness (CMT) outcomes. ${ }^{17-22}$ However, the efficacy of eplerenone therapy in CSC is still under investigation and needs further studies with randomized clinical trials.

In the last years, multimodal imaging provided a better understanding of pathophysiological mechanisms and of treatment outcomes of different retinal disease.

Nevertheless, no studies analyzed specific features at ICGA and at OCT-angiography (OCT-A) that could predict the final response to MR antagonists' treatment.

The aim of this study is to identify specific characteristics of treatment-naive patients affected by first onset of CSC with no rapid spontaneous resolution (within 2 months), by multimodal retinal imaging, in order to try to identify possible predictive biomarkers in case of treatment.

\section{Materials and methods}

The study is designed as an interventional, open-label, non-randomized clinical study in which all patients presenting at the Medical Retina \& Imaging Unit of the Department of Ophthalmology, University Vita-Salute, Ospedale San Raffaele in Milan (Italy) with a symptomatic treatment-naive CSC and presence of subretinal fluid (SRF) for $>2$ months were prospectively enrolled between November 2015 and April 2016. All patients in this study, which adhered to the tenets of the Declaration of Helsinki, signed a written consent, approved by the ethics committee of San Raffaele Hospital.

The criteria for inclusion were: (1) age greater than 18 years, (2) diagnosis of treatment-naive CSC with no spontaneous regression for at least 2 months, (3) presence of SRF involving the fovea on structural OCT.

The exclusion criteria were: (1) presence of cystic degeneration involving the fovea on structural OCT, (2) presence of any other retinal disorder potentially confounding the clinical assessment (eg, age-related macular disease, diabetic retinopathy, retinal vein occlusion, retinal artery occlusion), (3) myopia >6 diopters, (4) any previous treatment for CSC (eg, photodynamic therapy (PDT), anti-vascular endothelial growth factor intravitreal injection), (5) presence of significant media opacities (eg, cataract or corneal opacity), (6) presence of systemic contraindication for eplerenone treatment (liver or renal disease, hyperkalemia, defined as $>5.5 \mathrm{mmol} / \mathrm{l}$, or concomitant drugs that increase the potassium level, pregnancy). Patients with history of no spontaneous regression for at least 6 months and/or with other past episodes of CSC were considered as chronic CSC.

At baseline, all patients underwent a complete ophthalmologic examination, including BCVA, using Early Treatment Diabetic Retinopathy Study (ETDRS) charts, slit-lamp biomicroscopy, lens status examination, tonometry, indirect fundus ophthalmoscopy, infrared reflectance, fundus autofluorescence (FAF), structural spectral domain OCT (SD-OCT), fluorescein angiography (FA), ICGA, and OCT-A. Infrared reflectance, FAF, SDOCT, and ICGA were performed using Spectralis (Heidelberg Engineering, Heidelberg, Germany). FA was performed using Spectralis or TRC-50IX (Topcon, Inc., Tokyo, Japan). A 5-ml intravenous injection of 20\% fluorescein solution (Monico Spa, Italy) and $2.5 \mathrm{ml}$ of $5 \mathrm{mg} / \mathrm{ml}$ indocyanine green solution (Diagnostic Green $\mathrm{BmbH}$, Aschheim-Dornach, Germany) were injected to perform FA and ICGA, respectively.

Structural SD-OCT minimum acquisition protocol included: 19 horizontal raster linear B-scans, each composed by nine averaged OCT B-scans (1024A-scans per line) at $240 \mu \mathrm{m}$ intervals, covering an area of 20 degrees by 15 degrees; 6 radial linear B-scans, each composed by 25 averaged OCT B-scans (768A-scans per line) at 30 degrees centered on the fovea; 49 horizontal raster dense linear B-scans, each composed by 16 averaged OCT B-scans (384A-scans per line) at $30 \mu \mathrm{m}$ intervals, covering an area of 15 degrees by 5 degrees. To achieve a better visualization of the choroid, Enhanced Depth Imaging OCT was used in all acquisitions. CMT in the central 1-mm-diameter circle of the ETDRS thickness map was recorded with the Spectralis Software (Heidelberg Eye Explorer, Version 1.9.11.0 Heidelberg Engineering). SRF was assessed by manually measuring the distance between the end of the outer segment and the RPE at the foveal center. Subfoveal choroidal thickness (SFCT) was recorded by manually measuring the distance between the Bruch's membrane interface and the sclerochoroidal interface to identify the inner and outer boundaries of the choroid, respectively.

OCT-A examinations were performed using AngioPlex OCT angiographic imaging installed on the high definition OCT CIRRUS Model 5000 (Carl Zeiss Meditec, Dublin, CA, USA). To evaluate the morphology, we used a $3 \times 3 \mathrm{~mm}$ scanning area, which is composed by $245 \mathrm{~A}-$ scans in each B-scan along the horizontal direction and 245 B-scan positions along the vertical direction, and a $6 \times 6 \mathrm{~mm}$ scanning area, which is composed by 350Ascans in each B-scan along the horizontal direction and 350 B-scan positions along the vertical direction. Each B-scan is repeated four times in $3 \times 3$ area and twice in $6 \times 6$ area. All acquisitions were performed using FastTrac retinal-tracking technology to reduce motion artifacts. 
To determinate possible predictive biomarkers of response to therapy, we analyzed structural OCT, OCT-A, and ICGA images obtained at baseline. We selected four possible predictive biomarkers prior to the data analysis and we reviewed the results by using these biomarkers: presence of CNV at OCT-A, presence of CNV at ICGA, presence of punctate hyperfluorescent spot (hotspot) at ICGA and SFCT at structural OCT. The presence of CNV was selected as a possible predictive biomarker of response because was previously associated with worse prognosis in CSC patients; ${ }^{3}$ the presence of the hotspot at ICGA and the SFCT at structural OCT were selected as possible predictive biomarker because strictly related to the choroidal vascular hyperpermeability in CSC. ${ }^{7-10}$ All these features were evaluated by two trained examiners (AC and LQ), masked to each other. In case of discordance, the results were adjudicated by a senior retinal specialist (GQ).

Within current treatment options for CSC, all patients were treated by oral eplerenone (Inspra, Pfizer, Paris, France) to obtain a homogenous population. As reported in Supplementary Figure 1, the treatment regiment consisted in oral eplerenone $25 \mathrm{mg}$ /day for a week followed by $50 \mathrm{mg} /$ day for 4 weeks. All patients began the therapy after no spontaneous regression for at least 2 months and were revaluated at the end of 5-week therapy with complete ophthalmologic examination, including SD-OCT and OCT-A. The $50 \mathrm{mg}$ /day eplerenone treatment was continued for other 8 weeks depending on the presence of SRF at structural SD-OCT scans. All patients were revaluated at the end of the therapy and 8 weeks later. We considered a total reabsorption of SRF as a complete response to the treatment, and a reduction of at least $50 \%$ of SRF from baseline as a partial response.

Treatment tolerance was assessed by blood analyses of kaliemia and creatinine performed at baseline and every 4 weeks. The treatment was stopped in case of kaliemia increase $(>5 \mathrm{mmol} / \mathrm{l})$ or creatinine clearance rate decrease $(<60 \mathrm{ml} / \mathrm{min})$.

Primary outcomes included presence of pathological findings on ICGA, structural OCT, and OCT-A at baseline associated with different response to the treatment. Secondary outcomes included the evaluation of eplerenone effect, measured as the percentage of eyes achieving complete resolution of SRF on structural SDOCT after treatment, and changes in BCVA and CMT, also in patients further followed after the end of the protocol study.

Statistical analyses were performed using SPSS Statistics Version 20 (IBM, Armonk, New York, USA). Results of descriptive analyses are expressed as means \pm standard deviations for quantitative variables, and as counts and percentages for categorical variables. Association between categorical variables were analyzed using Fisher's Exact test; in detail, the following association were evaluated: between the presence of $\mathrm{CNV}$ at OCT-A and the complete response to the treatment, between the presence of CNV at ICGA and the complete response to the treatment, between the presence of the hotspot at ICGA and the response to the treatment and between the presence of a chronic CSC and the complete response to the treatment. Fisher's Exact test was also performed to test the interdependence between the biomarkers analyzed. The Gaussian distribution of continuous variables was verified with the KolmogorovSmirnov test. Comparisons of mean BCVA, CMT and SRF between baseline and the end of the treatment were performed using the Student paired $t$-test. Comparison of mean SFCT between patients with a complete response to the treatment and without and comparison of mean CSC duration between patients with $\mathrm{CNV}$ and without were performed using the Student independent samples $t$-test. In all analyses, $P$-values $<0.05$ were considered as statistically significant.

\section{Results}

A total of 29 eyes of 27 patients ( 2 females, 25 males) met the inclusion criteria and were included in the study. All patients were Caucasian and the mean age was $45 \pm 7$ years (median 48; range 29-56 years). Mean CSC duration at baseline was $13.5 \pm 4.4$ weeks (median 12; range 828 weeks). On structural SD-OCT, all patients presented $\mathrm{SRF}$ at baseline. The main clinical data at baseline are listed in Table 1A.

Table 1A Main changes in clinical parameters from baseline at each follow-up in all data

\begin{tabular}{|c|c|c|c|c|c|c|c|}
\hline & Baseline & 5-week examination & P-value & 13-week examination & P-value & 21-week examination & P-value \\
\hline BCVA, LogMAR (mean \pm SD) & $0.20 \pm 0.14$ & $0.12 \pm 0.13$ & $<0.001^{\mathrm{a}}$ & $0.10 \pm 0.11$ & $<0.001^{\mathrm{a}}$ & $0.9 \pm 0.12$ & $<0.001^{\mathrm{a}}$ \\
\hline $\mathrm{CMT}, \mu \mathrm{m}($ mean $\pm \mathrm{SD})$ & $398 \pm 107$ & $324 \pm 92$ & $0.001^{\mathrm{a}}$ & $298 \pm 83$ & $<0.001^{\mathrm{a}}$ & $288 \pm 71$ & $<0.001^{\mathrm{a}}$ \\
\hline $\mathrm{SRF}, \mu \mathrm{m}($ mean $\pm \mathrm{SD})$ & $166 \pm 93$ & $85 \pm 82$ & $<0.001^{\mathrm{a}}$ & $47 \pm 77$ & $<0.001^{\mathrm{a}}$ & $43 \pm 79$ & $<0.001^{\mathrm{a}}$ \\
\hline $\mathrm{SFCT}, \mu \mathrm{m}(\mathrm{mean} \pm \mathrm{SD})$ & $432 \pm 79$ & $422 \pm 81$ & $0.015^{\mathrm{a}}$ & $419 \pm 76$ & $0.008^{\mathrm{a}}$ & $411 \pm 81$ & $<0.001^{\mathrm{a}}$ \\
\hline
\end{tabular}

Abbreviations: BCVA, best-corrected visual acuity; CMT, central macular thickness; SD, standard deviation; SFCT, subfoveal choroidal thickness; SRF, subretinal fluid. ${ }^{\text {a }}$ Student paired $t$-test. 

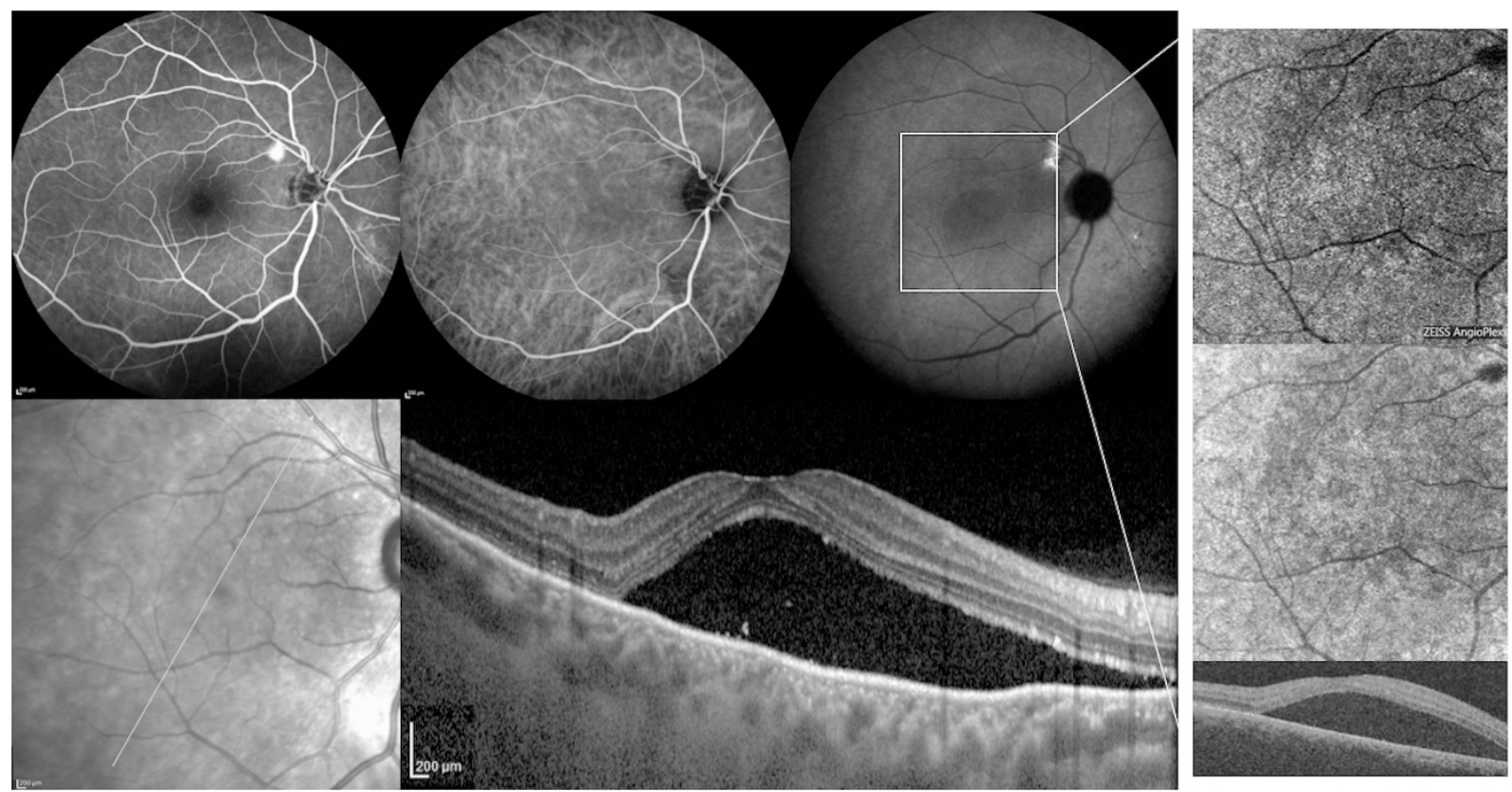

Figure 1 Fluorescein angiography (FA), indocyanine green angiography (ICGA), infrared reflectance (IR), optical coherence tomography (OCT), and OCT-angiography (OCT-A) of a patient affected by central serous chorioretinopathy (CSC) from 6 months. (Top row, first panel) FA at 4 min postinjection revealing a circular hyperfluorescence spot with 'ink-blot' pattern. (Top row, second and third panel) Early and late ICGA images showing no choroidal neovascularization (CNV) and the presence of a punctate hyperfluorescent spot (hotspot). (Bottom row, first and second panel) IR and structural OCT B-scan passing through the fovea showing the presence of a macular serous retinal detachment. (Top, middle, and bottom row, fourth panel) Choriocapillaris segmentation on $6 \times 6$ OCT-A, corresponding en-face OCT and B-scan with flow showing the absence of CNV.

At first follow-up visit, after 5 weeks of treatment, 9 out of 29 eyes (31\%) demonstrated complete SRF resolution on structural SD-OCT and thus discontinued therapy.

After 13 weeks (end of the treatment), among 29 eyes overall, 17 eyes (58\%) demonstrated complete SRF resolution on structural SD-OCT, 6 eyes $(21 \%)$ presented a partial response to eplerenone and 6 eyes (21\%) showed no substantial response (Figures 1,2,3,4). SRF decreased significantly compared with baseline (final SRF:

$47 \pm 77 \mu \mathrm{m}$, range $0-282 \mu \mathrm{m}, P<0.001)$. CMT decreased significantly from $398 \pm 107 \mu \mathrm{m}$ (range $219-596 \mu \mathrm{m}$ ) at baseline to $298 \pm 83 \mu \mathrm{m}$ (range 177-499 $\mu \mathrm{m} ; P<0.001$ ). Mean baseline BCVA improved to $\sim 20 / 25$ Snellen equivalent $(0.10 \pm 0.11 \mathrm{Log} M A R ;$ median 0.1 ; range $0-0.4$ LogMAR) at the end of treatment $(P<0.001)$ (Table 1A). Seven eyes (24\%) gained at least two ETDRS lines at the end of follow-up, 12 eyes (41\%) gained one ETDRS line, whereas BCVA worsening was recorded in one eye $(3 \%)$. Only one patient, who responded completely in the first 5 weeks and thus discontinued therapy, showed a partial relapse after 8 weeks. All other eyes showed no signs of relapse at the end of follow-up ( 8 weeks after the end of the treatment). Ten out of 17 eyes with a complete response to the therapy were further followed after the end of the protocol study for a total of $8.3 \pm 1.6$ months (range 6-12) after the treatment; 7 out of 10 cases $(70 \%)$ showed no signs of recurrence at the end of the follow-up, whereas 3 out of 10 cases (30\%) showed SRF

reappearance after a mean of $5.0 \pm 1.7$ months (range 3-6).

By analyzing FA images, we noticed two different patterns in patients affected by CSC. The majority of cases showed an 'ink-blot' or 'smokestack' pattern,

characterized by a hyperfluorescence arising from a central spot with or without dye diffusion; in other cases, generally characterized by the presence of $\mathrm{CNV}$ at multimodal imaging, there was a late-phase ill-defined hyperfluorescent lesion, without leakage or pooling of dye.

By evaluating the presence of CNV at OCT-A, we found that 8 out of 29 eyes showed CNV at baseline; 7 out of these 8 cases (87\%) also showed CNV at ICGA (Figures 3 and 4). There was a unanimous agreement on the presence of CNV between the two readers. On ICGA, $\mathrm{CNV}$ appeared as a hypercyanescent neovascular network in early-mid phases of ICGA, with wash-out effect in the late phase. On structural SD-OCT, CNVs were characterized by a flat irregular elevation of the RPE with moderately reflective material in the sub-RPE space. The complete response to eplerenone treatment was associated with absence of CNV at OCT-A and ICGA $(P<0.001$ and $P=0.001$, respectively). None of eight eyes with presence of CNV in OCT-A imaging had a complete 


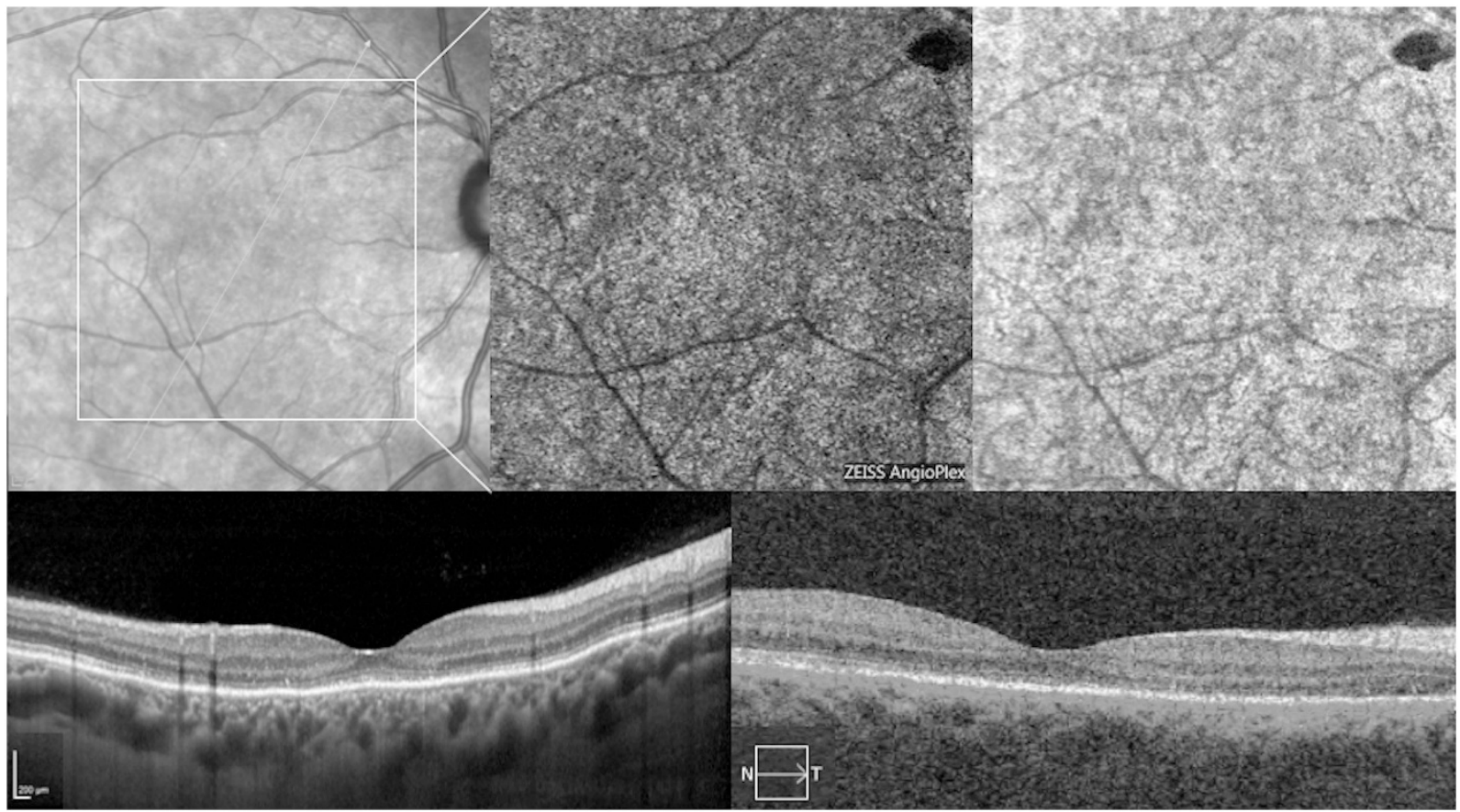

Figure 2 Infrared reflectance (IR), optical coherence tomography (OCT), and OCT-angiography (OCT-A) of the same patient of Figure 1 at 13-week follow-up. (First panel) IR and structural OCT B-scan passing through the fovea showing the resolution of macular serous retinal detachment. (Second and third panel) Choriocapillaris segmentation on $6 \times 6$ OCT-A, corresponding en-face OCT and B-scan with flow showing yet the absence of CNV.

resolution after eplerenone treatment, and only four out of eight eyes (50\%) showed improvement (Figures 3 and 4). Seventeen eyes out of 21 eyes without evidence of CNV (81\%) demonstrated complete SRF resolution on structural SD-OCT after eplerenone treatment, 2 eyes $(9.5 \%)$ presented a partial response to treatment, and 2 eyes $(9.5 \%)$ showed no substantial response (Table $1 \mathrm{~B})$. No statistical difference in mean CSC duration was found between patients with $\mathrm{CNV}$ and without CNV $(13.0 \pm 3.5$ versus $14.9 \pm 6.3$ weeks, respectively, $P=0.304$ ).

Furthermore, the presence of a chronic form of CSC was not associated with lower rate of response to the treatment; 11 out of 18 eyes (61\%) affected by chronic CSC had a complete response to eplerenone, compared to 6 out of 11 eyes $(55 \%)$ without a chronic CSC $(P=0.514)$. Considering only 8 eyes with CNV on OCT-A and no response to eplerenone therapy, 4 were subsequently treated by half-fluence PDT with complete anatomical response.

By evaluating the presence of hotspot at ICGA, 26 out of 29 eyes (90\%) showed a punctate hyperfluorescent spot at baseline and this finding was associated with favorable response to eplerenone treatment $(P=0.002)$ (Table $1 \mathrm{~B})$. This spot was detected in all eyes without CNV at OCT-A, and in four out of eight (50\%) eyes affected by $\mathrm{CNV}$ showing a significant correlation between the presence of a CNV and the presence of the hotspot at ICGA
$(P=0.015)$. Four out of five eyes with CNV and ICGA hotspot had a partial response to eplerenone treatment, whereas none of three eyes with CNV and without hotspot showed a significant improvement at the end of therapy $(P=0.028)$.

Finally, no difference was disclosed in SFCT between patients with a complete response to treatment and without (mean SFCT: $424 \pm 85 \mu \mathrm{m}$ and $444 \pm 70 \mu \mathrm{m}$, respectively $(P=0.517))$.

These findings suggest that both presence of $\mathrm{CNV}$ at OCT-A/ICGA and absence of hotspot at ICGA may be considered as predictive biomarkers of unfavorable response to the treatment.

\section{Discussion}

In this study, we investigated potential predictors at OCT$A$ and ICGA imaging that could be associated with different response to eplerenone therapy. The efficacy of eplerenone still remains to be proven in further randomized investigations and was not the primary outcome of this study.

During the last decades, several treatments have been proposed for CSC. Half-fluence and half-dose PDT represent the most effective treatment for chronic CSC, but are associated with risks of porphyrin-induced phototoxic dermal reaction, potential photoreceptor 

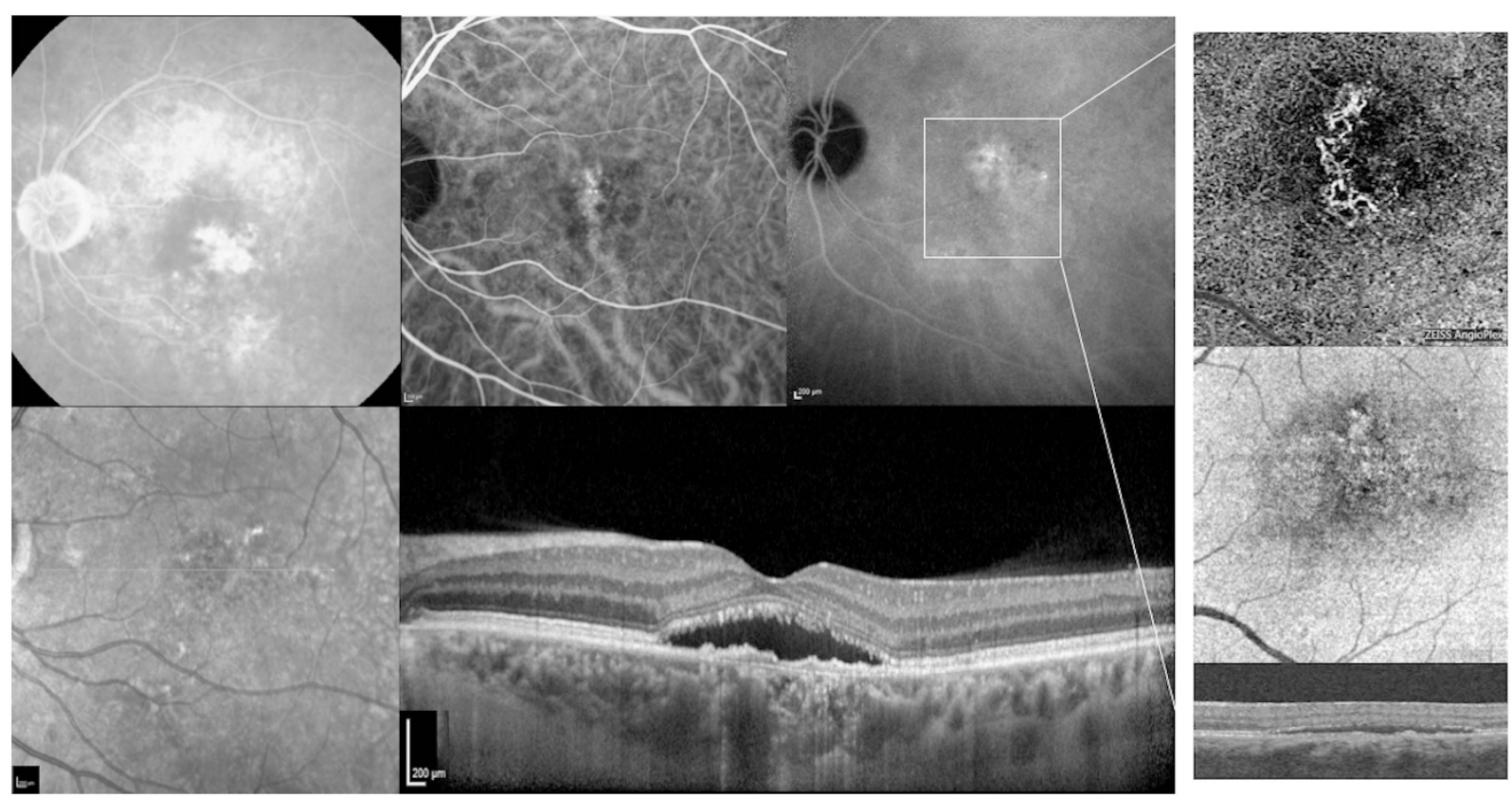

Figure 3 Fluorescein angiography (FA), indocyanine green angiography (ICGA), infrared reflectance (IR), optical coherence tomography (OCT), and OCT-angiography (OCT-A) of a patient affected by central serous chorioretinopathy (CSC) from 4 months. (Top row, first panel) FA at $5 \mathrm{~min}$ postinjection revealing a late-phase ill-defined hyperfluorescent lesion, without leakage or pooling of dye. (Top row, second and third panel) Early and late ICGA images showing a hyperfluorescent area corresponding to the choroidal neovascularization $(\mathrm{CNV})$ along with punctate hyperfluorescent spots. (Bottom row, first and second panel) IR and structural OCT B-scan passing through the fovea showing the presence of a macular serous retinal detachment and the presence of a flat irregular elevation of the RPE with moderately reflective material in the sub-RPE space, compatible with a type $1 \mathrm{CNV}$. (Top, middle and bottom row, fourth panel) Choriocapillaris segmentation on $3 \times 3$ OCT-A, corresponding en-face OCT and B-scan with flow showing the presence of $\mathrm{CNV}$.

disruption, alterations of the RPE or neurosensory retina, and secondary choroidal neovascularization. ${ }^{23-26}$

To the best of our knowledge, no studies have been published about predictors of response to therapy for CSC using ICGA and OCT-A. Only Cakir and associates ${ }^{22}$ using structural OCT and FAF reported that patients with integrity of the RPE and ellipsoid zone were associated with a favorable BCVA response to oral eplerenone. We believe that a definition of predictive biomarkers is useful to identify patients that most likely can benefit from treatment. Among the 29 eyes enrolled, 8 eyes showed presence of CNV using OCT-A and in seven of these eight cases CNV was confirmed by ICGA, showing a great agreement between these two techniques. OCT-A is a useful non-invasive tool to detect and assess the morphology of CNV in many retinal disease including age-related macular degeneration. ${ }^{27-29}$ Moreover, our data suggest that OCT-A could be used more safely and effectively as an alternative of ICGA to detect presence of $\mathrm{CNV}$ in CSC.

Subgroup analysis in these patients showed that $>90 \%$ of patients without $\mathrm{CNV}$ had a complete or partial resolution of SRF on structural SD-OCT after treatment (Figures 1 and 2), whereas no patients with CNV had a complete resolution of SRF and four out of eight patients had only a partial response (Figures 3 and 4). This statistically significant difference $(P<0.001)$ demonstrated that presence of CNV at OCT-A can be considered an important unfavorable predictor of response to eplerenone treatment. One can argue that different response to the treatment between patients affected by $\mathrm{CNV}$ and without CNV may be caused by possible different duration of the disease. However, in CSC patients affected by $\mathrm{CNV}$ we did not find the duration of the disease to be significantly longer than in patients without CNV $(13.0 \pm 3.5$ versus $14.9 \pm 6.3$ weeks, patients with and without $\mathrm{CNV}$, respectively $(P=0.304))$.

Furthermore, our results suggested that also the presence of a chronic form of CSC was not associated with lower rate of response to the treatment $(P=0.586)$. For these reasons, we do not believe that the duration of the disease may have affected the different response between groups.

Our results indicated also that presence of hotspot at ICGA imaging was associated with a difference in eplerenone therapy response $(P=0.002)$. Tsujukawa and associates $^{30}$ and Park and associates ${ }^{31}$ reported that these punctate hyperfluorescent spots at ICGA may be a manifestation of the increased choroidal hyperpermeability and intrachoroidal hydrostatic pressure. Interestingly, considering the subgroup of eight 


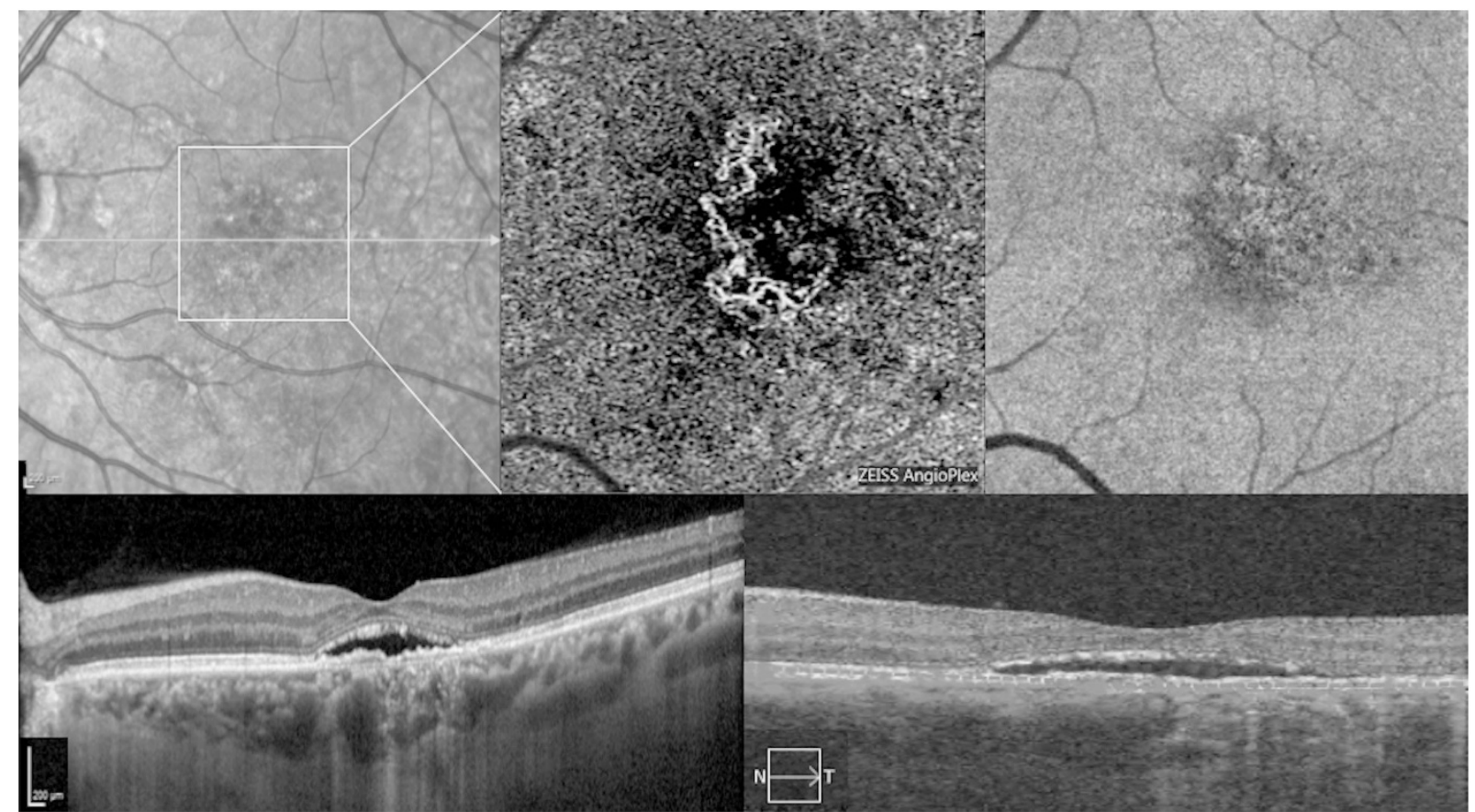

Figure 4 Infrared reflectance (IR), optical coherence tomography (OCT), and OCT-angiography (OCT-A) of the same patient of Figure 3 at 13-week follow-up. (First panel) IR and structural OCT B-scan passing through the fovea showing the persistence of macular serous retinal detachment and the presence of a hyperreflective area between the Bruch's membrane and the irregularly elevated retinal pigment epithelium. (Second and third panel) Choriocapillaris segmentation on $3 \times 3$ OCT-A, corresponding en-face OCT and B-scan with flow showing still the persistence of CNV.

Table 1B Subgroup analysis of anatomical response to eplerenone therapy at 13-week follow-up

\begin{tabular}{|c|c|c|c|c|c|c|c|}
\hline & \multirow{2}{*}{$\begin{array}{l}\text { All patients } \\
(\mathrm{n}, 29)\end{array}$} & \multicolumn{2}{|c|}{$\begin{array}{c}\text { Presence of } C N V \\
\text { at OCT-A }\end{array}$} & \multicolumn{2}{|c|}{$\begin{array}{c}\text { Presence on } C N V \\
\text { at ICGA }\end{array}$} & \multicolumn{2}{|c|}{$\begin{array}{c}\text { Presence of hotspot } \\
\text { at ICGA }\end{array}$} \\
\hline & & Yes $(\mathrm{n}, 8)$ & No $(n, 21)$ & Yes $(\mathrm{n}, 7)$ & No $(\mathrm{n}, 22)$ & Yes $(\mathrm{n}, 26)$ & No $(n, 3)$ \\
\hline \multicolumn{8}{|c|}{ Anatomical response (SRF reabsorption) at 13-week follow-up } \\
\hline Complete & $17(58 \%)$ & $0(0 \%)$ & $17(81 \%)$ & $0(0 \%)$ & $17(77 \%)$ & $17(65 \%)$ & $0(0 \%)$ \\
\hline Partial & $6(21 \%)$ & $4(50 \%)$ & $2(9.5 \%)$ & $4(57 \%)$ & $2(9 \%)$ & $6(23 \%)$ & $0(0 \%)$ \\
\hline No & $6(21 \%)$ & $4(50 \%)$ & $2(9.5 \%)$ & $3(43 \%)$ & $3(14 \%)$ & $3(12 \%)$ & $3(100 \%)$ \\
\hline
\end{tabular}

Abbreviations: CNV, choroidal neovascularization; OCT-A, optical coherence tomography angiography; ICGA, indocyaine green angiography; SRF, subretinal fluid.

eyes with $\mathrm{CNV}$, the presence of hotspot could be useful to identify patients that most likely benefit from treatment. Indeed, four out of five eyes (80\%) with both CNV and hotspot at ICGA imaging had a partial response while no eye affected by CNV without hotspot at ICGA showed significant improvement at the end of therapy $(P=0.028)$.

Based on these observations, we could speculate that there are three possible typologies of treatment-naive CSC patients:

- Patients characterized by absence of CNV and presence of hotspot, in which SRF is likely due only to an increased choroidal hyperpermeability, thus responding almost constantly and completely to treatment;
- Patients characterized by presence of both CNV and hotspot, in which SRF is probably due to both, a manifestation of increased choroidal hyperpermeability and $\mathrm{CNV}$ itself, thus responding only partially to treatment;

- Patients characterized by presence of $\mathrm{CNV}$ but without hotspot, in which SRF is likely due only to the $\mathrm{CNV}$ itself and thus not responding to treatment.

We acknowledge that this study has several limitations, mainly owing to the absence of a placebo-treated group, the relatively small sample size and the relative short duration of follow-up. Nevertheless, in our series, eplerenone therapy seems to provide significant anatomical and functional improvement in 
selected treatment-naive patients affected by CSC. We further followed 10 eyes with a complete response to the treatment after the end of the protocol study, and found a recurrence rate of $30 \%$.

In conclusion, using multimodal imaging we reported for the first time the presence of potential predictive biomarkers that are very useful for identifying patients that most likely may respond to treatment. Using the presence of CNV at OCT-A and of hotspot at ICGA as prognostic biomarkers, it seems possible to have a better selection of patients to treat by eplerenone therapy, although further studies with larger sample size are needed to confirm our results. Given that the efficacy of eplerenone was not the primary outcome of this study, further randomized investigations should be performed to demonstrate the beneficial effects of MR antagonists in CSC.

\section{Summary}

What was known before

- No studies analyzed specific biomarkers at ICGA and at OCT-A that could predict the final response to MR antagonists' treatment in CSC.

\section{What this study adds}

- We found that using the presence of choroidal neovascularization at OCT-A and of hotspot at ICGA as prognostic biomarkers, it seems possible to have a better selection of patients to treat by eplerenone therapy.

\section{Conflict of interest}

The authors declare no conflict of interest.

\section{Acknowledgements}

Riccardo Sacconi, Giovanni Baldin, Adriano Carnevali, Lea Querques, Alessandro Rabiolo: none. Giorgio Marchini consultant for: Alcon (Fort Worth, TX, USA), Allergan Inc (Irvine, CA, USA), Bausch and Lomb (Rochester, NY, USA), Santen (Osaka, Japan). Francesco Bandello consultant for: Alcon (Fort Worth, TX, USA), Alimera Sciences (Alpharetta, GA, USA), Allergan Inc (Irvine, CA, USA), Farmila-Thea (Clermont-Ferrand, France), Bayer Shering-Pharma (Berlin, Germany), Bausch And Lomb (Rochester, NY, USA), Genentech (San Francisco, CA, USA), Hoffmann-La-Roche (Basel, Switzerland), NovagaliPharma (Évry, France), Novartis (Basel, Switzerland), Sanofi-Aventis (Paris, France), Thrombogenics (Heverlee, Belgium), Zeiss (Dublin, USA). Giuseppe Querques consultant for: Alimera Sciences (Alpharetta, GA, USA), Allergan Inc (Irvine, CA, USA), Heidelberg (Germany), Novartis (Basel, Switzerland),
Bayer Shering-Pharma (Berlin, Germany), Zeiss (Dublin, USA). None of the Authors received financial or material support for the research and the work from any of the following organizations: National Institutes of Health (NIH); Wellcome Trust; Howard Hughes Medical Institute (HHMI); and other(s).

\section{References}

1 Kitzmann AS, Pulido JS, Diehl NN, Hodge DO, Burke JP. The incidence of central serous chorioretinopathy in Olmsted County, Minnesota,1980-2002. Ophthalmology 2008; 115: 169-173.

2 Quin G, Liew G, Ho IV, Gillies M, Fraser-Bell S. Diagnosis and interventions for central serous chorioretinopathy: review and update. Clin Exp Ophthalmol 2013; 41: 187-200.

3 Loo RH, Scott IU, Flynn HW Jr, Gass JD, Murray TG, Lewis ML et al. Factors associated with reduced visual acuity during long-term follow-up of patients with idiopathic central serous chorioretinopathy. Retina 2002; 22: 19-24.

4 Guyer DR, Yannuzzi LA, Slakter JS, Sorenson JA, Ho A, Orlock D. Digital indocyanine green videoangiography of central serous chorioretinopathy. Arch Ophthalmol 1994; 112: 1057-1062.

5 Prünte C, Flammer J. Choroidal capillary and venous congestion in central serous chorioretinopathy. Am J Ophthalmol 1996; 121: 26-34.

6 Liew G, Quin G, Gillies M, Fraser-Bell S. Central serous chorioretinopathy: a review of epidemiology and pathophysiology. Clin Exp Ophthalmol 2013; 41: 201-214.

7 Iida T, Kishi S, Hagimura N, Shimizu K. Persistent and bilateral choroidal vascular abnormalities in central serous chorioretinopathy. Retina 1999; 19: 508-512.

8 Jirarattanasopa P, Ooto S, Tsujikawa A, Yamashiro K, Hangai M, Hirata $\mathrm{M}$ et al. Assessment of macular choroidal thickness by optical coherence tomography and angiographic changes in central serous chorioretinopathy. Ophthalmology 2012; 119: 1666-1678.

9 Imamura Y, Fujiwara T, Margolis R, Spaide RF. Enhanced depth imaging optical coherence tomography of the choroid in central serous chorioretinopathy. Retina 2009; 29: 1469-1473.

10 Kim SW, Oh J, Kwon SS, Yoo J, Huh K. Comparison of choroidal thickness among patients with healthy eyes, early age-related maculopathy, neovascular age-related macular degeneration, central serous chorioretinopathy, and polypoidal choroidal vasculopathy. Retina 2011; 31: 1904-1911.

11 Bouzas EA, Karadimas P, Pournaras CJ. Central serous chorioretinopathy and glucocorticoids. Surv Ophthalmol 2002; 47: 431-448.

12 Tittl MK, Spaide RF, Wong D, Pilotto E, Yannuzzi LA, Fisher YL et al. Systemic findings associated with central serous chorioretinopathy. Am J Ophthalmol 1999; 128: 63-68.

13 Carvalho-Recchia CA, Yannuzzi LA, Negrão S, Spaide RF, Freund $\mathrm{KB}$, Rodriguez-Coleman $\mathrm{H}$ et al. Corticosteroids and central serous chorioretinopathy. Ophthalmology 2002; 109: 1834-1837.

14 Haimovici R, Rumelt S, Melby J. Endocrine abnormalities in patients with central serous chorioretinopathy. Ophthalmology 2003; 110: 698-703. 
15 van Dijk EH, Schellevis RL, van Bergen MG, Breukink MB, Altay L, Scholz P et al. Association of a haplotype in the NR3C2 gene, encoding the mineralocorticoid receptor, with chronic central serous chorioretinopathy. JAMA Ophthalmol 2017; 135: 446-451.

16 Zhao M, Célérier I, Bousquet E, Jeanny JC, Jonet L, Savoldelli $\mathrm{M}$ et al. Mineralocorticoid receptor is involved in rat and human ocular chorioretinopathy. J Clin Invest 2012; 122: 2672-2679.

17 Bousquet E, Beydoun T, Zhao M, Hassan L, Offret O, Behar-Cohen F. Mineralocorticoid receptor antagonism in the treatment of chronic central serous chorioretinopathy: a pilot study. Retina 2013; 33: 2096-2102.

18 Breukink MB, den Hollander AI, Keunen JE, Boon CJ, Hoyng CB. The use of eplerenone in therapy-resistant chronic central serous chorioretinopathy. Acta Ophthalmol 2014; e488-e490.

19 Leisser C, Hirnschall N, Hackl C, Plasenzotti P, Findl O. Eplerenone in patients with chronic recurring central serous chorioretinopathy. Eur J Ophthalmol 2016; 26: 479-484.

20 Chin EK, Almeida DR, Roybal CN, Niles PI, Gehrs KM, Sohn $\mathrm{EH}$ et al. Oral mineralocorticoid antagonists for recalcitrant central serous chorioretinopathy. Clin Ophthalmol 2015; 11: 1449-1456.

21 Ghadiali Q, Jung JJ, Yu S, Patel SN, Yannuzzi LA. Central serous chorioretinopathy treated with mineralorticoid antagonists: a one-year pilot study. Retina 2016; 36: 611-618.

22 Cakir B, Fischer F, Ehlken C, Bühler A, Stahl A, Schlunck G et al. Clinical experience with eplerenone to treat chronic central serous chorioretinopathy. Graefes Arch Clin Exp Ophthalmol 2016; 254: 2151-2157.

23 Chan WM, Lam DS, Lai TY, Tam BS, Liu DT, Chan CK. Choroidal vascular remodelling in central serous chorioretinopathy after indocyanine green guided photodynamic therapy with verteporfin: a novel treatment at the primary disease level. Br J Ophthalmol 2003; 87: 1453-1458.

24 Lim JI, Glassman AR, Aiello LP, Chakravarthy U, Flaxel CJ, Spaide RF Macula Society CSC Collaborative Study Group. Research and Education Committee and Website Committee. Collaborative retrospective macula society study of photodynamic therapy for chronic central serous chorioretinopathy. Ophthalmology 2014; 121: 1073-1078.

25 Ratanasukon M, Thongthong K, Bhurayanontachai P, Jirarattanasopa P. Photoreceptor disruption in central serous chorioretinopathy treated by half-dose photodynamic therapy. Clin Ophthalmol 2013; 7: 87-92.

26 Colucciello M. Choroidal neovascularization complicating photodynamic therapy for central serous retinopathy. Retina 2006; 26: 239-242.

27 De Carlo TE, Bonini Filho MA, Chin AT, Adhi M, Ferrara D, Baumal CR et al. Spectral-domain optical coherence tomography angiography of choroidal neovascularization. Ophthalmology 2015; 122: 1228-1238.

28 Coscas GJ, Lupidi M, Coscas F, Cagini C, Souied EH. Optical coherence tomography angiography versus traditional multimodal imaging in assessing the activity of exudative age-related macular degeneration: a new diagnostic challenge. Retina 2015; 35: 2219-2228.

29 Miere A, Querques G, Semoun O, El Ameen A, Capuano V, Souied EH. Optical coherence tomography angiography in early type 3 neovascularization. Retina 2015; 35: 2236-2241.

30 Tsujikawa A, Ojima Y, Yamashiro K, Ooto S, Tamura H, Nakagawa $\mathrm{S}$ et al. Punctate hyperfluorescent spots associated with central serous chorioretinopathy as seen on indocyanine green angiography. Retina 2010; 30: 801-809.

31 Park SJ, Kim BH, Park KH, Woo SJ. Punctate hyperfluorescence spot as a common choroidopathy of central serous chorioretinopathy and polypoidal choroidal vasculopathy. Am J Ophthalmol 2014; 158: 1155-1163.

Supplementary Information accompanies this paper on Eye website (http://www.nature.com/eye) 